\title{
Courting Apocalypse: Creating a Zombie-Themed Evidence-Based Medicine
}

\author{
Game \\ Amy E. Blevins \\ Elizabeth Kiscaden \\ Jason Bengtson
}

\begin{abstract}
In 2015, two librarians at the Hardin Library for the Health Sciences at the University of Iowa turned their dreams into a reality and secured funding to build a zombiethemed evidence-based medicine game. The game features a "choose your own adventure" style that takes students through a scenario where a disease outbreak is taking place and a resident is asked to use evidence-based medicine skills to select a screening and diagnostic tool to use on potentially infected patients. Feedback on the game has been positive, and future plans include building additional modules on therapy, harm, and prognosis.
\end{abstract}

KEYWORDS. Evidence-based medicine, games, gamification, medical education, zombies

\section{Authors.}

Amy E. Blevins, MALS (blevinsamy@gmail.com) is Associate Director for Public Services, Ruth Lilly Medical Library, Indiana University School of Medicine, 975 W. Walnut St., Indianapolis, IN 46202.

Elizabeth Kiscaden, MLIS, AHIP (elizabeth-kiscaden@uiowa.edu) is Associate Director, Greater Midwest Region, National Network of Library of Medicine, Hardin Library for the Health Sciences, 600 Newton Road, Iowa City, IA 52242. 
Jason Bengtson, MLIS, MA (jbengtson@ksu.edu) is Assistant Director, IT Services, Kansas

State Libraries, Kansas State University, 414 Hale Library, Manhattan, KS 66506.

Amy Blevins and Elizabeth Kiscaden both worked at Hardin Library for the Health Sciences at the University of Iowa during the creation of this game.

This project was presented as a lightning talk at the Medical Library Association conference in

Toronto, Ontario, Canada on May 15, 2016 and as a paper at the Merge \& Converge: Sixteen in

'16 Joint Chapter Meeting (Midwest/MCMLA) in Des Moines, IA on October 23, 2016.

\section{Acknowledgements}

Special thanks go out to Anna Kramer who produced the artwork for this game; Dawn Ebach, M.

D., who consulted with the creators on the story and quiz elements of the game; and Helen

Spielbauer, who used her photography and graphic design skills to put the final images together.

This game would not have been possible without your contributions. 


\section{INTRODUCTION}

Gaming has become increasingly prevalent in medical education, as illustrated by numerous cases in the published literature. Gaming as an educational tool in the health sciences has advanced to teach students and health professionals increasingly complex clinical competencies. Recent examples of advanced educational games used in the health sciences include those designed to: teach emergency care skills, ${ }^{1}$ organize pills for patients to prevent medication errors, ${ }^{2}$ adapt to equipment problems in the surgical theater, ${ }^{3}$ and determine the appropriate insulin therapy for patients. ${ }^{4}$

Within the medical education curriculum, gaming has become increasingly more established as projects have been designed and validated by research. At a training center at Heidelberg University, medical students between their third and sixth year participated in a game intended to provide instruction on correct chest tube insertion, ${ }^{5}$ which has recently been the subject of a randomized controlled trial. At the National University of Singapore, over 200 Phase III medical students used an interactive iPad game to learn about patient safety issues in surgery, ${ }^{6}$ which resulted in progressively improved understanding of patient safety. A game designed to improve understanding of a concept as complex as laparoscopic cholecystectomy has even been validated with medical students and found to be realistic and useful in learning. ${ }^{7}$

Teaching evidence-based medicine (EBM) concepts to medical students via interactive games is not entirely uncharted territory, although there are few examples available in the literature. One notable project involved a collaboration between librarians at the University of Washington education staff at the School of Medicine to develop an online game called Research and Evidence Learning in Medicine (RELM). ${ }^{8}$ RELM was designed to use medical cases to 
teach students how to ask answerable clinical questions and locate evidence to answer their question, two important pillars of evidence-based medicine that involve library instruction. RELM is not currently under development by the University of Washington Health Sciences Library but is publicly accessible at http://relm.hsl.washington.edu:3001/.

Recognizing an opportunity to build off the evidence-based medicine gaming concept pioneered by the University of Washington, librarians at the University of Iowa partnered with faculty and a developer to create a proof-of-concept zombie game named “Zombies Ate My Evidence: The EBM Challenge to Save the World” at http://www.lib.uiowa.edu/hardin/zombiesate-my-evidence/. The team was able to secure limited funding for this project through the University of Iowa Libraries’ Innovation Fund in June of 2015, which made this project possible.

\section{BACKGROUND}

The University of Iowa Carver College of Medicine has designed a series of flipped classroom sessions to teach their students evidence-based medicine concepts. The majority of the content is delivered in the pre-clerkship portion of the curriculum. During clerkships, students are asked to apply their skills in the clinical settings to answer questions that arise from patient cases. Therefore, this game was designed as a fun way to reinforce the skills taught in the pre-clerkship curriculum to assist students with critically appraising articles. The game could also be used by students as a self-assessment of EBM skills, but was not designed with sufficient explanations to serve as a teaching tool.

The team hired a student artist from the University of Iowa to develop illustrated characters for the game, and began the initial work of building a storyboard. A pediatrics 
gastroenterologist and EBM enthusiast had worked with the designers on several classes for medical students in the pediatrics clerkship and assisted with the clinical scenarios and EBM concept. One of the library assistants at Hardin Library took the artwork from the student artist and combined it with photographs she took around Iowa City to create the final images for the game.

The design of the game was that of a choose-your-own-adventure style story, in which a young resident was assisting with an outbreak at her hospital. Within the story, the resident uses her evidence-based medicine skills to choose a screening and diagnostic tool, and is pointed to modules available online for review. As the game was designed as a proof-of-concept only, the story is left at a cliffhanger, leaving room for future expansion.

Tapping into the popularity of zombies in the media, the outbreak portrayed within the game was intentionally similar to zombie television shows, such as The Walking Dead. As such, the game was titled “Zombies Ate My Evidence: The Evidence-Based Medicine Challenge to Save the World!” Characters drawn by the artist were superimposed upon photos of actual locations within the University of Iowa Hospitals and Clinics in order to create a sense of place for medical students playing the game. Figure 1is a screenshot of the opening scene for the game where Sassy receives a call from her boyfriend, Bran, while on call.

[INSERT FIGURE 1 HERE]

\author{
Legend: FIGURE 1. Opening Scene for Zombies Ate My Evidence
}

STORYLINE AND INSTRUCTIONAL ELEMENTS 
The opening scene for this game shows the protagonist, a resident named Sassy, receiving a call from her boyfriend, a paramedic named Bran, while she's on call at the hospital. It turns out that there's an outbreak in the city and patients are being quarantined. Students viewing the game are enlisted to help Sassy pick the best screening tool by reviewing three different tests. For each test, the students are provided with a description of how the test works and information on the number of patients tested that were known to have the disease, the number of patients tested that were known to be without the disease, the number of true positives, and the number of true negatives. The students have to use the skills they have acquired through previous EBM modules to calculate the specificity and sensitivity of each test. They then have to use their knowledge of screening tests to pick the best one to use. If the students answer the quiz question incorrectly, they are referred back to the diagnosis module to review the content and try again. If they get the answer correct, they proceed with the game. In Figure 2, the player chooses the wrong response and zombies overrun the city.

\section{[INSERT FIGURE 2 HERE]}

\section{Legend: FIGURE 2. Zombies Take Over the City}

Once a screening test is chosen, Sassy is asked to find a diagnostic test to confirm that patients are infected to keep the hospitals quarantine from being overrun. In the second quiz, Sassy is given the prevalence of the disease and a pre-test probability. Several tests are offered as options. This quiz is meant to remind students of the usefulness of likelihood ratios and nomograms to move from an uncertain pre-test probability to a more certain post-test probability. Similar to the previous quiz question, if the student gives a wrong response, the student is 
prompted to review the diagnosis EBM module before attempting the quiz again. If the student answers correctly, the game continues.

The reference standards presented earlier in the game include a brain biopsy that is fast but very risky. The other "test” involved quarantining patients for 14 days, since most patients died and reanimated within seven to ten days with a few outliers. This information was included because it allows Sassy to have a conversation with her attending about patient values. He explains to Sassy that some patients are willing to wait a longer time or undergo a riskier test due to different values. The game ends with Sassy discovering that her boyfriend, Bran, has been infected with the virus, and the cliffhanger is that she needs to investigate potential treatment options in the next module. In Figure 3, Sassy is in full quarantine gear and her boyfriend, Bran, is strapped to a hospital bed beginning to exhibit the classic signs of zombie-ism.

\section{[INSERT FIGURE 3 HERE]}

\section{Legend: FIGURE 3. Bran is in quarantine.}

\section{PROGRAMMING DETAILS}

The project developer was recruited to the team because of his work on another, similar game, and his experience building complex applications. Not all libraries possess the technical infrastructure to create a project like this. Nor do all libraries have the funds and contacts necessary to outsource such expertise, as on this case. However, for those that do, some additional detail may not only be of general interest, but may help them as they approach the 
decisions needed for such a project. These details are useful to help readers appreciate the technical complexity of such an undertaking.

The application for this game was built entirely in HTML, CSS, and Javascript, to execute in the browser (known as the "client-side”). The decision was made to make the game more lightweight and portable, and was possible because the game used a limited amount of data. In contrast to this, many web applications are built using server-side scripting with a relational database to store the data, leaving part of the application's code to be executed on the server. While a client-side only mode of construction carries some unique advantages, it also limits the ability of the game to perform in multiplayer modes, or to save game data anywhere but in limited local data stores.

Providing user interface elements, such as dialog boxes, accordion menus, and buttons was a challenge, particularly as the interface was developed alongside the storyboarding process. The developer used the jQuery UI library, one of the most popular user interface libraries available. jQuery UI has proven to be robust, broadly cross-browser compatible, and visually appealing.

In addition to jQuery UI, there are many frameworks and libraries which make the coherent design of an application such as this one both easier and more logical. One popular framework for this purpose is Google’s AngularJS. Through the use of AngularJS 1.0, a Javascript framework, much of the interactivity of the application could be developed by adding a series of additional "attributes” directly to HTML elements (a feature known in AngularJS as a “directive”). ${ }^{9}$

Most modern web applications are built using some variation of a design pattern known as Model-View-Controller (MVC). This pattern separates the application into three layers: one 
containing the data (model), one which interacts with the user (view), and one which negotiates between the other two layers (controller). There are many possible flavors of MVC, such as Model-View-Presenter, but these flavors follow the same general philosophy. ${ }^{10}$ This application was organized into a roughly MVC model with the AngularJS controller residing in a single file where the scenes and quiz data were stored as Javascript Object Notation (JSON) objects, making them easy to edit or expand with any text editor, rather than requiring a separate tool to allow them to be edited in a database on a server. AngularJS delivered this data dynamically to the HTML file of the application through the mechanism of directives. In this way, AngularJS, and, more importantly, the application’s main Javascript file, functioned as the actual control layer.

The choose-your-own-adventure design of the game is reflected in the structure of the game data, which was serialized in JSON format and stored as part of the AngularJS controller file. Each scene is serialized as a discrete object with a set of properties, including a call to the background image file. The two quizzes within the game were encoded in much the same way. When the game is played and the game files are loaded into the browser, special "ng-” prefixed attributes in elements in the HTML file are used as markers by the AngularJS framework, causing the JSON data to be populated into a series of hidden HTML < div> elements in the presentation layer of the application. The player moves through these $<$ div $>$ elements as they move through the game.

Rather than design the quizzes as scenes of the storyboard, the developer used "modal" $<$ div > elements, which are similar in appearance to pop-up boxes (although they use a different mechanism and are unaffected by pop-up blockers) and which required the player to solve them before proceeding with the story. Modals are a special type of “dialog” element which can be 
easily generated using the jQuery UI Javascript library. ${ }^{11}$ Fired by "trigger” attributes attached to particular scenes in the storyboard, this format allowed for a particularly flexible application of the quiz engine. With this model, quizzes were serialized separately in the JSON data and did not have to be added in a particular order with the other scenes.

This lightweight, flexible structure will allow for expansion of the game; a consideration as this project was a proof-of-concept only. Because this game was built exclusively with HTML, CSS, and Javascript (client-side web code), another benefit of this design was the elimination of the need for any special server setup. Essentially, the files could be hosted and run from any web-accessible server - a strategy the game’s developer had used in a previous project. ${ }^{12}$ The team gained support from the information technology team at the University of Iowa Libraries’ department to host the game on their server.

\section{TESTING/FEEDBACK}

The link for the game was sent to medical educators, medical students, and physician assistants. Along with the link to the game, there was a link to a short online survey. The creators received very limited, but useful, feedback. Some quotes included: "needs more gore”; "like the UIHC setting”; "Love the zombies, seems applicable!”; "great artwork!”; "Lots of unnecessary dialog.”

Despite minimal promotion, the game has been viewed over 443 times with 343 unique users between April 2016 and May 2017. The University of Iowa Carver College of Medicine had an entering class of 152 students in $2016 .{ }^{13}$ After this game was presented at a library conference in October 2016, a librarian from another institution reached out to the creators to say 
that she was using the game with her students. She provided the following feedback and suggestions for future development.

$<\mathrm{EX}>$

"We like the idea of the game being a place that we can send students to practice their EBM stats calculations. We have calculations for specificity and sensitivity calculations on their EBM I exam. They are also on Step I. The zombie focus makes it fun to practice stats (not an easy task). Students are always asking for more practice questions too. We also used the tutorial for a couple of in-class examples. ... I think any way to make those EBM concepts easier to learn would be helpful. I would love someone to create a tool that focused on statistical power and how expected effect size affects how many individuals you need in a study.”14

$</ \mathrm{EX}>$

\section{FUTURE IMPROVEMENTS}

From a design perspective, the application is something of an experiment, and feedback from others using the game or interested in contributing to the game is solicited. The narrative of the game should be continued, and a scoring mechanism for quizzes could be added. Along with scoring, future developments may include a server-side component for purposes such as storing high scores. In addition, it may be advantageous to create an app or mobile-friendly version of the game.

Although feedback on the game was mostly positive and enthusiastic, feedback did indicate there was a bit too much story. In the future, care will be taken to minimize the narrative 
and to include more interactive elements. Future modules would include therapy, etiology/risk, and prognosis. It is yet to be seen if the character in the story will recover from the zombie virus. This game could also be expanded to cover issues surrounding shared decision-making and infectious diseases.

Along with opportunities for improvement and expansion, this game offers some interesting opportunities for promotion. The project team has discussed loading the game onto USB drives and distributing it as a marketing tool at library events on campus. The library’s branding on the drive may send a message about the technological sophistication of the library and provide patrons with a new way to engage.

\section{CONCLUSION}

Overall, the game has been a fun project and has been a success according to its creators. While the three main game designers have changed jobs, there is still a strong interest in finishing additional modules in the future. The development of the game gave the creators a chance to build on existing EBM skills while creating the quiz questions. The final product allows medical students to test their EBM skills in a low stakes situation, and the zombie-themed game aspects provide entertainment value. Promoting the game allows the library to demonstrate that librarians are partners in EBM education with an interest in gamification and other newer instructional methods. This game serves as one example of gamification as a tool for students to self-assess learning. There are many other ways that librarians could use gamification to teach and assess EBM, information literacy, and other topics in the future. 
Received: May 24, 2017

Revised: July 7, 2017

Accepted:

\section{REFERENCES}

1. Dankbaar, Mary E. W., Maartje B. Roozeboom, Esther A. P. B Oprins, et al. "Preparing Residents Effectively in Emergency Skills Training with a Serious Game.” Simulation in Healthcare: The Journal of the Society for Simulation in Healthcare 12, no. 1 (February 2017): 9-16.

2. Bukowski, Mark, Martin Kühn, Xiaoqin Zhao, et al. "Gamification of Clinical Routine: The Dr. Fill Approach.” Studies in Health Technologies and Informatics 225 (2016):262-266.

3. Graafland, Maurits, Willem A. Bemelman, and Marlies P. Schijven. "Game-Based Training Improves the Surgeon's Situational Awareness in the Operation Room: A Randomized Controlled Trial.” Surgical Endoscopy (March 9, 2017): Epub ahead of print.

4. Diehl, Leandro A., Rodrigo M. Souza, Juliana B. Alves, et. al. "InsuOnline, an Electronic Game for Medical Education on Insulin Therapy: A Randomized Controlled Trial with Primary Care Physicians.” Journal of Medical Internet Research 19 no. 3. (March 9, 2017): e72.

5. Friedrich, Mirco, Christian Bergdolt, Patrick Haubruck, et al. “App-based Serious Gaming for Training of Chest Tube Insertion: Study Protocol for a Randomized Controlled Trial.” Trials 18, no. 1. (February 6 2017): 56. 
6. Kow, A. W. C., B. L. S. Ang, C. S. Chong, et al. "Innovative Patient Safety Curriculum Using iPAD Game (PASSED) Improved Patient Safety Concepts in Undergraduate Medical Students.” World Journal of Surgery 40 no. 11 (November 2016): 2571-2580.

7. Kowalewski, Karl-Friedrich, Jonathan D. Hendrie, Mona W. Schmidt, et al. "Validation of the Mobile Serious Game Application Touch Surgery ${ }^{\mathrm{TM}}$ for Cognitive Training and Assessment of Laparoscopic Cholecystectomy.” Surgical Endoscopy (March 9, 2017): Epub ahead of print.

8. Gleason, Ann. W. "RELM: Developing a Serious Game to Teach Evidence-Based Medicine in an Academic Health Sciences Setting.” Medical Reference Services Quarterly 34 no 1. (January-March 2015): 17-28.

9. Google (USA). “AngularJS: Developer Guide: Directives.” Accessed May 22, 2017. https://docs.angularjs.org/guide/directive.

10. Qureshi, M. Rizwan, and Fatima Sabir. “A Comparison of Model View Controller and Model View Presenter.” Science International 25 no. 1 (2013): 7-9.

11. The jQuery Foundation (USA). “The jQuery Foundation (USA).” Accessed May 22, 2017. https://jqueryui.com/dialog/\#modal-for.

12. Bengtson, Jason. “Funding a Gamification Machine.” In Library Technology Funding, Planning, and Development, edited by Stephen F. Austin, 99-112. Hershey: IGI Global, 2016.

13. University of Iowa. "Carver College of Medicine, MD Program: 2016 Entering Class Profile.” Accessed June 26, 2017. https://medicine.uiowa.edu/md/admissions/requirements/2016-entering-class-profile.

14. McEwen, Heather. Email communication to author. January 252017. 